05,01

\title{
Взаимодействие Дзялошинского-Мория в синтетических ферримагнетиках $\mathrm{Pt} / \mathrm{Co} / \mathrm{Ir} / \mathrm{Co} / \mathrm{Pt}$
}

\author{
(ㄱ А.И. Безверхний ${ }^{1}$, В.А. Губанов ${ }^{2}$, А.В. Садовников ${ }^{2}$, Р.Б. Моргунов ${ }^{1, \text { ॠ }}$ \\ ${ }^{1}$ Институт проблем химической фризики РАН, \\ Черноголовка, Россия \\ ${ }^{2}$ Саратовский национальный исследовательский университет, \\ Саратов, Россия \\ ฯ E-mail: morgunov2005@yandex.ru
}

Поступила в Редакцию 19 мая 2021 г.

В окончательной редакции 2 августа 2021 г.

Принята к публикации 3 августа 2021 r.

$\mathrm{B}$ синтетических ферримагнетиках $\mathrm{Pt} / \mathrm{Co} / \mathrm{Ir} / \mathrm{Co} / \mathrm{Pt}$ с перпендикулярной магнитной анизотропией обнаружена невзаимность спиновых волн методом мандельштам-бриллюэновской спектроскопии (МБС). Показано, что основной вклад в невзаимность спиновых волн вносит взаимодействие Дзялошинского-Мория с плотностью энергии $D \approx 1.7-2.3 \mathrm{erg} / \mathrm{cm}^{2}$. Плотность энергии взаимодействия Дзялошинского-Мория в синтетических ферримагнетиках $\mathrm{Pt} / \mathrm{Co} / \mathrm{Ir} / \mathrm{Co} / \mathrm{Pt}$ больше, чем ее значения в однослойных гетероструктурах $\mathrm{Pt} / \mathrm{Co}(1 \mathrm{~nm}) / \mathrm{Ir} \sim 1.4 \mathrm{erg} / \mathrm{cm}^{2}$ и уменьшается с ростом толщины незакрепленного слоя Со.

Ключевые слова: синтетические ферримагнетики, перпендикулярная магнитная анизотропия, рассеяние Мандельштама-Бриллюэна, обменное взаимодействие, взаимодействие Дзялошинского-Мория.

DOI: $10.21883 /$ FTT.2021.12.51665.120

\section{1. Введение}

Многослойные гетероструктуры и сверхрешетки представляют интерес в области изучения спиновых волн $(\mathrm{CB})$, так как они являются основой для устройств антиферромагнитной спинтроники [1]. В первых работах, посвященных исследованию СВ методом мандельштамбриллюэновской спектроскопии (МБС) в структурах спинтоники $[2,3]$, объектом исследования были гетероструктуры, состоящие из двух ферромагнитных слоев с легкими осями намагничивания в плоскости, разделенных слоем немагнитного проводящего вещества (FM/NM/FM). Метод МБC представляет собой спектроскопию упругого рассеяния света на собственных колебаниях твердого тела с изменением частоты рассеянного фотона. Рассеяние может происходить, как на фононах, так и на спиновых волнах (магнонах). Максимумы на спектрах МБС соответствуют частотам фононов и спиновых волн, на которых произошло рассеяние фотона.

На частотных спектрах МБС одиночных тонких ферромагнитных пленок наблюдаются два пика рассеяния на спиновых волнах: пик Стокса и пик анти-Стокса [4]. Эти пики соответствуют СВ, распространяющимся в тонких пленках в направлениях, противоположных друг другу и ортогональных направлению эффективного магнитного поля. В многослойных гетероструктурах FM/NM/FM, состоящих из двух обменно-связанных ферромагнитных слоев, спектры МБС могут отличаться от спектров одиночных ферромагнитных пленок наличием двух пиков Стокса и двух пиков анти-Стокса [3], что яв- ляется проявлением дисперсии СВ в обменно-связанных ферромагнитных слоях. При изменении толщины прослойки между слоями ферромагнетика меняется энергия $J_{12}$ межслоевого обменного взаимодействия Ruderman-Kittel-Kasuya-Yosida (RKKY). В образцах $\mathrm{Fe} / \mathrm{Au} / \mathrm{Fe}$ и $\mathrm{Fe} / \mathrm{Cr} / \mathrm{Fe}[3]$ было обнаружено изменение частоты одного пика Стокса и одного пика анти-Стокса спиновых волн при изменении $J_{12}$. Частоты остальных пиков не зависели от $J_{12}$. Обменное взаимодействие между спинами, разделенными прослойкой ферромагнитных слоев, приводит к согласованному распространению спиновых волн в них, что может быть представлено в виде суперпозиции акустической и оптической ветвей спектра. Частоты спиновых волн, не зависящих от $J_{12}$, принадлежат акустической моде. Акустическая мода ассоциируется со связанными синфазными спиновыми волнами с одинаковыми значениями $k$ в двух ферромагнитных слоях (рис. 1). Оптическая мода вызвана межслоевым антиферромагнитным обменным взаимодействием между спинами в ферромагнитных слоях. В результате антиферромагнитного обмена между спинами они прецессируют в противофазе [2,5].

Еще одним следствием взаимодействия между ферромагнитными слоями является разница $\Delta f$ между частотами пика Стокса $f(k)$ и пика анти-Стокса $f(-k)$. Эта разница частот зависит от поверхностной анизотропии двух ферромагнитных слоев [6], от дипольного взаимодействия и от энергии антисимметричного обменного взаимодействия Дзялошинского-Мория (DMI) [7], возникающего на границах разделов ферромагнетика и тяжелого металла с сильным спин-орбитальным взаи- 


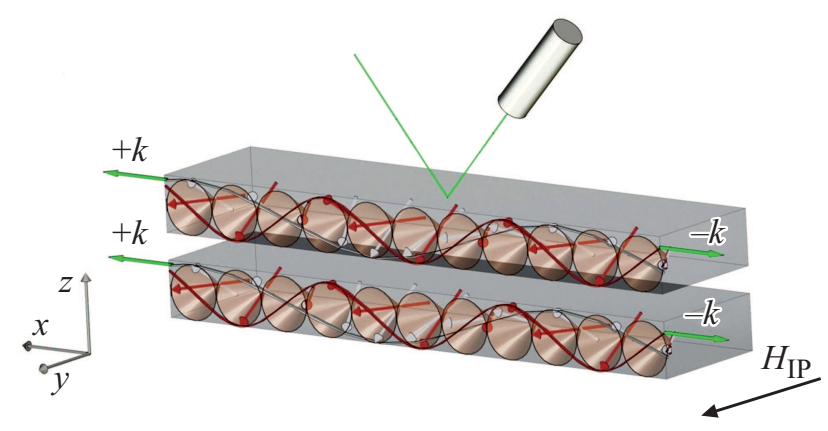

Pис. 1. Спиновые волны в синтетическом антиферромагнетике в постоянном внешнем магнитном поле $H_{\mathrm{IP}}$.

модействием [8]. В литературе разницу частот пиков Стокса и анти-Стокса $\Delta f$ (невзаимность спиновых волн) часто ошибочно воспринимают, как однозначное свидетельство наличия DMI и используют ее для измерения плотности энергии DMI $-D$. Хотя метод МБС является мощным инструментом прямого измерения энергии DMI, для его использования нужно учитывать и другие источники невзаимности частот пиков Стокса и анти-Стокса.

Основное количество работ по измерению плотности энергии DMI методом МБС сделано в гетероструктурах на основе одиночных ферромагнитных пленок, но интерес представляет усложнение этой ситуации в структуpax FM/NM/FM за счет обменного смещения, межслоевого обмена RKKY и разницы констант поверхностной анизотропии двух ферромагнитных слоев. Например, в [9] были проведены аккуратные измерения МБС в образцах $\mathrm{Co} / \mathrm{Au} / \mathrm{Co}$ и $\mathrm{Co} / \mathrm{Cu} / \mathrm{Co}$, в которых толщины $\mathrm{Co}$ были одинаковы, а толщина прослойки внутри одного образца изменялась ступенчато с шагом $0.4 \mathrm{~nm}$. Таким образом, авторы [9] смогли корректно учесть влияние поверхностной и магнитокристаллической анизотропии на спектр спиновых волн в слоях Со. Это позволило точно измерить методом МБС энергию межслоевого обмена $J_{12}$, как функцию только толщины прослоек $\mathrm{Au}$ и $\mathrm{Cu}$, исключив вклад поверхностной анизотропии. В результате была обнаружена знакопеременная осцилляция энергии межслоевого обменного взаимодействия при изменении толщины прослойки. Позднее, тем же методом, была обнаружена осцилляция обменной межслоевой энергии в сверхрешетках $[\mathrm{Co} / \mathrm{Ru}]_{20}[10]$.

Из литературы известно, что на границах раздела $\mathrm{Pt} / \mathrm{Co}$ и $\mathrm{Pt} / \mathrm{Ir}$ в гетероструктурах $\mathrm{Pt} / \mathrm{Co} / \mathrm{Pt}[11,12]$, $\mathrm{Pt} / \mathrm{Co} / \mathrm{Ir} / \mathrm{Pt}$ [13] и $\mathrm{Pt} / \mathrm{Co} / \mathrm{Ir}$ [14] наблюдается DMI c плотностью энергии $D \sim 1 \mathrm{erg} / \mathrm{cm}^{2}$. В исследуемых нами образцах $\mathrm{Pt} / \mathrm{Co} / \mathrm{Ir} / \mathrm{Co} / \mathrm{Pt}$ также можно ожидать наличие DMI, поскольку в образцах имеются интерфейсы Pt/Co и $\mathrm{Pt} / \mathrm{Ir}$. В литературе широко представлены работы по изучению DMI в гетероструктурах на основе одиночной пленки Со [11-14], в то время как в обменносвязанных ферромагнитных слоях DMI малоизученно. Цель статьи заключается в изучении спиновой динамики методом МБС в синтетических ферримагнетиках (СФ) $\mathrm{Pt} / \mathrm{Co} / \mathrm{Ir} / \mathrm{Co} / \mathrm{Pt}$, в которых сочетается антиферромагнитное обменное взаимодействие между слоями Со и DMI на интерфейсах $\mathrm{Pt} / \mathrm{Co}$ и $\mathrm{Pt} / \mathrm{Ir}$.

\section{2. Образцы и методика экспериментов}

Исследуемые образцы являются многослойными гетероструктурами $\mathrm{SiO} 2 / \operatorname{Pt}(3.2 \mathrm{~nm}) / \mathrm{Co}(1.1 \mathrm{~nm}) / \mathrm{Ir}(1.4 \mathrm{~nm}) /$ $\mathrm{Co}\left(t_{\mathrm{Co}}\right) / \mathrm{Pt}(3.2 \mathrm{~nm})$, где $t_{\mathrm{Co}}-$ толщина верхнего слоя Со равна $0.6,0.7,0.8$ и $1.0 \mathrm{~nm}$. Образцы размером $2 \times 3 \mathrm{~mm}$ получены методом магнетронного напыления при комнатной температуре в сверхвысоком вакууме $\left(10^{-8}\right.$ Torr). Слои Со обладают перпендикулярной магнитной анизотропией вследствие гибридизации атомных $5 d$-орбиталей Со с орбиталями Pt и Ir [15]. Подробные исследования магнитных свойств и динамики перемагничивания этих образцов были представлены в работах $[16,17]$, где были установлены энергии анизотропии слоев, обменные взаимодействия между слоями, критические поля переключения намагниченности и другие свойства.

Петли магнитного гистерезиса при ориентациях внешнего магнитного поля вдоль поверхности образца $\left(H_{\mathrm{IP}}\right)$ и перпендикулярно ей $\left(H_{\mathrm{OP}}\right)$ были записаны СКВИД магнетометром MPMS 5XL Quantum Design.

Для измерения спектров рассеяния света методом МБС при комнатной температуре была использована геометрия Дэмона-Эшбаха и конфигурация обратного рассеяния, т.е. внешнее поле $H_{\mathrm{IP}}$ было направленно в плоскости пленки перпендикулярно волновому вектору СВ и перпендикулярно плоскости падения света (см. рис. 1). Измерения проводились при углах падения лазерного луча $15,30,45$ и $60^{\circ}$. Этим углам соответствуют проекции волнового вектора света, падающего на поверхность образца, равные $k_{\mathrm{x} 1}=7 \mu \mathrm{m}^{-1}, k_{\mathrm{x} 2}=11 \mu \mathrm{m}^{-1}$, $k_{\mathrm{x} 3}=16 \mu \mathrm{m}^{-1}$ и $k_{\mathrm{x} 4}=20 \mu \mathrm{m}^{-1}$. В качестве источника света использовался лазерный луч с длинной волны $532 \mathrm{~nm}$, генерируемый одночастотным лазером Excelsior (Spectra Physics) EXLSR-532-200-CDRH. Диаметр сфокусированного на поверхности образца лазерного пятна составлял $25 \mu \mathrm{m}$. Мощностью лазерного излучения $20 \mathrm{~mW}$ была достаточно мала, чтобы не приводить к нагреву образца. Важно отметить, что эффективная глубина проникновения лазерного луча составляет 30-40 nm [18], что превышает суммарную толщину слоев исследуемых гетероструктур. Таким образом, все данные полученные методом МБС вызваны суммарным вкладом всех слоев. Разницы частот пиков Стокса и анти-Стокса были определены из измерений, проведенных во внешнем постоянном магнитном поле напряженностью $+8 \mathrm{kOe}$. Это внешнее поле близко к эффективному полю анизотропии, определенному из рис. 2. Величины этого поля было достаточно для ориентации спинов в плоскости образца. Запись спектров МБС является процессом длительного накопления сигнала. 


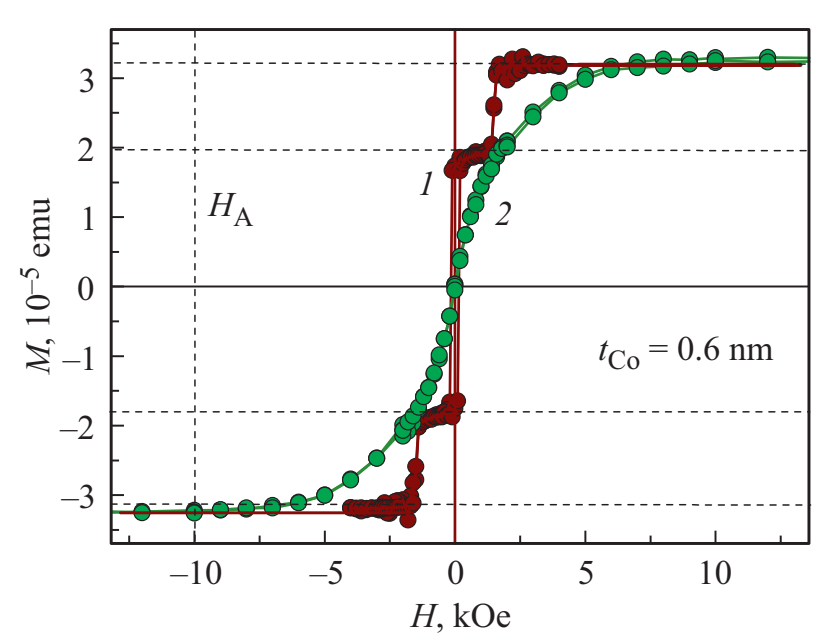

Рис. 2. Полевые зависимости намагниченности $\mathrm{M}(\mathrm{H})$ образца c $t_{\mathrm{Co}}=0.6 \mathrm{~nm}$, записанные при направлении поля перпендикулярно (1) и параллельно (2) плоскости образца. Штриховыми горизонтальными линиями показаны равновесные состояния намагниченности образца в поле, направленном нормально к поверхности образца. Вертикальной штриховой линией показано поле эффективной магнитной анизотропии $H_{\mathrm{A}}=10 \mathrm{kOe}$.

Время накопления на исследуемых в настоящей статье образцах составляло 10-12 h. За это время производилось в среднем 100 измерений для каждой частоты в интервале от $-30 \mathrm{GHz}$ до $+30 \mathrm{GHz}$ с шагом $125 \mathrm{MHz}$.

Математическое моделирование дисперсий спиновых волн $f\left(k_{\mathrm{x}}\right)$ и зависимости частоты спиновых волн от межслоевой обменной энергии $f\left(J_{12}\right)$ было проведено с использованием программного обеспечения Wolfram Mathematica 12.1.

\section{3. Результаты и обсуждение}

На спектрах МБС у образцов с $t_{\text {Со }}=0.7,0.8$ и $1.0 \mathrm{~nm}$ наблюдались по одному пику Стокса и одному пику анти-Стокса (рис. $3, a-3, c)$. На спектре образца с $t_{\text {Со }}=0.6 \mathrm{~nm}$ (рис. $3, d$ ) наблюдалось два пика Стокса и два пика анти-Стокса. Аналогичные сдвоенные спектры наблюдали в $[2,3,6,7]$. Два пика, частоты которых не зависят от энергии межслоевого обмена, принадлежат акустической моде СВ. Спиновые волны, частоты которых зависят от межслоевого обмена, принадлежат оптической моде. По соотношению интенсивности пиков Стокса и анти-Стокса можно судить о соотношении между количествами актов рассеяния фотонов на СВ с векторами $+k_{\mathrm{x}}$ (Стокс) и (анти-Стокс) $-k_{\mathrm{x}}$. Количество актов рассеяния на СВ зависит от времени жизни соответствующих магнонов [19]. Соотношение интенсивностей пиков Стокса и анти-Стокса (см. рис. 3) для всех образцов было примерно одинаковым $I_{S} / I_{A S} \sim 1.18$. Следовательно, отношение среднего времени жизни магнонов $\mathrm{c}+k_{\mathrm{x}}$ к среднему времени жизни магнонов с про- тивоположным волновым вектором $k_{\mathrm{x}}$ было одинаковым во всех образцах.

Чтобы убедиться в том, что наличие двух пиков Стокса и двух пиков анти-Стокса на спектре МБС образца с $t_{\text {Со }}=0.6 \mathrm{~nm}$ вызвано оптической и акустической модами, зависимость частот спиновых волн $f$ от величины обменной межслоевой энергии $J_{12}$ моделировали в соответствии с моделью, предложенной в [20]. В отличие от модели, представленной в [21] для однослойных структур, в [20] предложены расчеты для описания синтетических антиферромагнетиков и ферримагнетиков с перпендикулярной магнитной анизотропией. Для моделирования были выбраны следующие параметры: одинаковое поле кубической анизотропии нижнего и верхнего слоев Со $H_{\mathrm{ac} 1}=H_{\mathrm{ac} 2} \approx 152 \mathrm{Oе}[22]$, одинаковое поле одноосной анизотропии слоев Со $H_{\mathrm{au} 1}=H_{\mathrm{au} 2} \approx 384 \mathrm{Oе}$, обменная жесткость Со $A=1.6 \cdot 10^{-6} \mathrm{erg} / \mathrm{cm}$, намагниченность насыщения пленки Со $M_{\mathrm{S}}=1300 \mathrm{emu} / \mathrm{cm}^{3}$ [23], гиромагнитное отношение Со $\gamma=1.9 \cdot 10^{7} \mathrm{~Hz} / \mathrm{Oe}$ [24]. Волновой вектор спиновых волн был постоянным $k_{\mathrm{x}}=11 \mu \mathrm{m}^{-1}$. Поле поверхностной анизотропии слоев Со $H_{\mathrm{ac} 1} \approx H_{\mathrm{ac} 2} \approx 1400$ Ое было определено из данных СКВИД-магнитометрии. Из полевых зависимостей намагниченности, записанных при ориентации внешнего поля перпендикулярно поверхности образца (рис. 2 кривая 1) и вдоль нее (рис. 2 кривая 2), следует, что в поле $H_{\mathrm{IP}}=+8 \mathrm{kOe}$ намагниченность $M \approx M_{\mathrm{S}}$. Внешнее магнитное поле $H_{\mathrm{IP}}=+8 \mathrm{kOe}$, направленное в плоскости образца, ориентирует магнитные моменты вдоль поля. Таким образом, углы между осью $у$ и магнитными моментами составляют $\theta_{1}=\theta_{2} \approx 0^{\circ}$. Так как образцы обладают перпендикулярной магнитной анизотропией, угол между полем $H_{\mathrm{IP}}$ и направлением магнитной анизотропии $\theta_{H}=90^{\circ}$, а углы между осью у и направлением одноосной анизотропии двух слоев Со равны $\theta_{\mathrm{u} 1}=\theta_{\mathrm{u} 2}=90^{\circ}$. Результат моделирования зависимости частоты спиновых волн от энергии межслоевой обменной связи $J_{12}$ показан на рис. 4 сплошными линиями. Сплошной линией 1 на рис. 4 показана акустическая мода, а сплошной линией 2 оптическая мода. Точками на рис. 4 показаны экспериментальные частоты стоксовой составляющей спектров МБС, записанные в постоянном поле $H_{\mathrm{IP}}=+8 \mathrm{kOe}$ при постоянном $k_{\mathrm{x}}=11 \mu \mathrm{m}^{-1}$. В отсутствии межслоевого обмена $\left(J_{12}=0\right)$, частоты оптической и акустической мод близки по значению $[3,25,26]$. На рис. 4 треугольником показана частота спиновой волны образца $\mathrm{Pt} / \mathrm{Co}(1.07 \mathrm{~nm}) / \mathrm{Ir}$ [27], у которого $J_{12}=0$, так как он содержит один ферромагнитный слой. Обменная энергия $J_{12}$ между разделенными слоями ферромагнетика зависит не только от толщины разделяющей прослойки [9], но и от толщины ферромагнитных слоев [28]. В [28] показано, что зависимость $J_{12}$ от толщины одного из ферромагнитных слоев может осциллировать, т. е. характер этой зависимости аналогичен зависимости $J_{12}$ от толщины разделяющей прослойки [9,28]. Это происходит потому, что в проводящем слое Со при уменьшении 

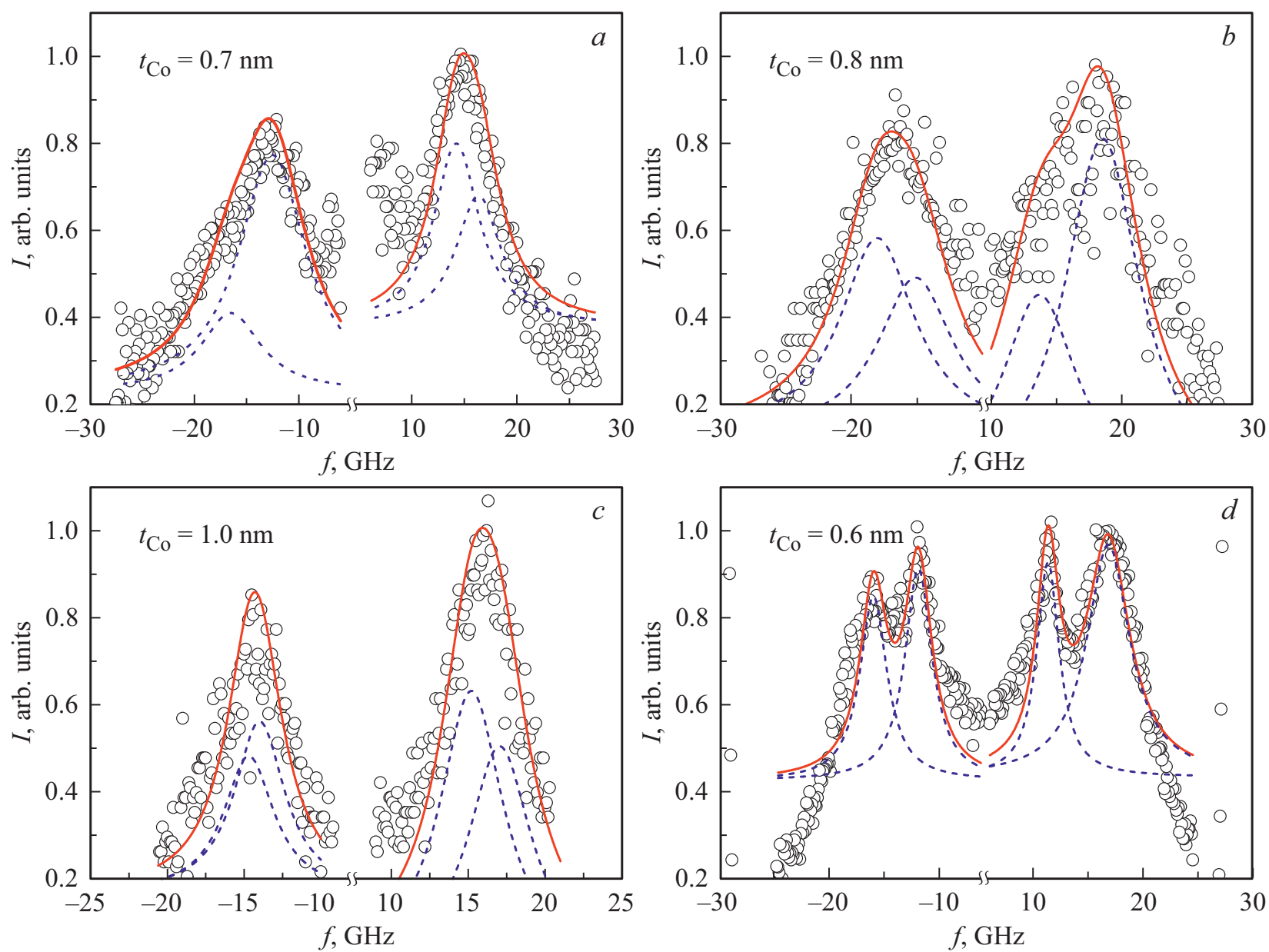

Рис. 3. Спектры мандельштам-бриллюэновской спектроскопии, записанные в поле $H_{\mathrm{IP}}=+8 \mathrm{kOe}$ при значении проекции волнового вектора лазерного луча $k_{\mathrm{x}}=11 \mu \mathrm{m}^{-1}$ для образцов с $(a) t_{\mathrm{Co}}=0.7 \mathrm{~nm},(b) t_{\mathrm{Co}}=0.8 \mathrm{~nm},(c) t_{\mathrm{Co}}=1.0 \mathrm{~nm},(d) t_{\mathrm{Co}}=0.6 \mathrm{~nm}$. Пунктирные линии - это лоренцевы компоненты разложения экспериментального спектра на оптическую и акустическую моды, описанные в тексте. Сплошные линии на рис. $3, a-d-$ это сумма лоренцевых линий.

его толщины, уменьшается количество атомов, и этот ферромагнитный слой становится менее эффективным в качестве источника спин-поляризованных электронов, участвующих в RKKY взаимодействии. Таким образом, величина $J_{12}$ в серии исследуемых образцов меняется вместе с толщиной верхнего слоя Со. Обменная межслоевая энергия $J_{12}$ между слоями Со в исследуемых образцах посчитана из данных СКВИД-магнитометрии. Методика расчета $J_{12}$ приведена в [16]. Толщины $t_{\text {Со, }}$ соответствующие значениям $J_{12}$ образцов, показаны на верхней шкале рисунка 4. Частоты пиков на МБС спектрах были определены путем их аппроксимации функциями Лоренца методом наименьших квадратов. Погрешность определения частот использована для обозначения погрешностей на рис. 4 и 5. Разница погрешностей частот для разных образцов вызвана разной скоростью и качеством накопления сигнала. Скорость и качество накопления сигнала зависели не только от образца, но и от угла падения лазерного луча, а следовательно от величины $k_{\mathrm{x}}$.
Результат моделирования частоты оптической и акустической мод совпадает с экспериментальными значениями частот $\mathrm{CB}$ образца с $t_{\mathrm{Co}}=0.6 \mathrm{~nm}$. Теоретический расчет зависимости частоты оптической моды от межслойного обменного взаимодействия хорошо согласуется с экспериментальными значениями частот СВ для образцов с $t_{\text {Со }}=0.7 \mathrm{~nm}, 0.8 \mathrm{~nm}, 1.0 \mathrm{~nm}$. Для последних трех образцов малая разница между оптической и акустической модами приводит к слиянию этих двух пиков. Поскольку частота акустической моды не зависит от $J_{12}[2,3]$, для всех образцов она должна быть примерно одинаковая. Она точно определена для того образца, где пики оптической и акустической ветви разделены ( 16.6 GHz). Это облегчало аппроксимацию пиков двумя функциями Лоренца в тех образцах, с $t_{\mathrm{Co}}=0.7$, $0.8,1.0 \mathrm{~nm}$, где пики акустической и оптической моды сливались. Результат разложения спектра на два пика Лоренца показан на рис. $3, a-d$ штриховыми линиями. Сумма двух функций Лоренца показана на рис. 3, $a-c$ сплошными линиями. Установленные аппроксимацией 


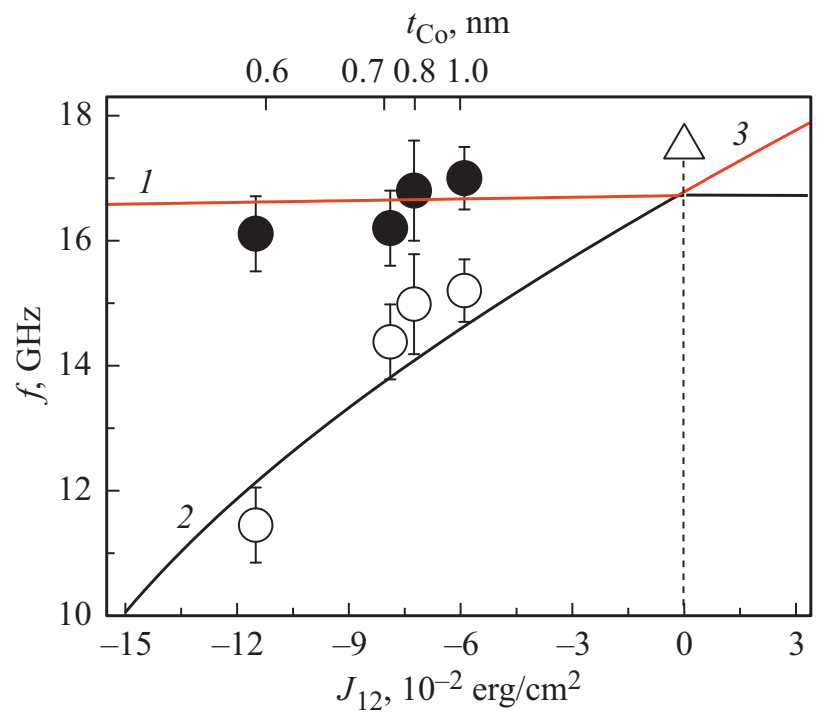

Рис. 4. Зависимость частоты спиновых волн от межслоевой обменной энергии $J_{12}$ в поле $H_{\mathrm{IP}}=+8 \mathrm{kOe}$ при $k_{\mathrm{x}}=11 \mu \mathrm{m}^{-1}$. Светлыми символами показаны частоты стоксова пика оптической моды, а темными символами - частоты стоксова пика акустической моды. Точка 3 - частота спиновой волны $\left(k_{\mathrm{x}}=11 \mu \mathrm{m}^{-1}\right)$ для образца, состоящего из одного слоя Со $\left(J_{12}=0\right)$ [26]. Сплошные линии теоретические зависимости частоты акустической моды (линия 1) и оптической моды (линия 2) от межслоевой обменной энергии, рассчитанные в рамках модели, предложенной в [14]. Толщины верхнего слоя Со, соответствующие указанным значениям $J_{12}$, приведены на верхней шкале.

частоты двух мод для образцов с $t_{\mathrm{Co}}=0.6,0.7,0.8$, $1.0 \mathrm{~nm}$ показаны на рис. 4 точками.

На всех спектрах МБС образцов $\mathrm{Pt} / \mathrm{Co} / \mathrm{Ir} / \mathrm{Co} / \mathrm{Pt}$ была обнаружена разница абсолютных значений частот пиков Стокса и анти-Стокса $\Delta f$, которую называют невзаимностью спиновых волн [2]. Для пиков акустической моды невзаимность $\Delta f$ частот обычно не наблюдается, и график дисперсии спиновых волн $f\left(k_{\mathrm{x}}\right)$ является параболой, симметричной относительно $k_{\mathrm{x}}=0$ (см. рис. 5,a). Разница частот обычно возникает для оптических мод и может быть вызвана разницей поверхностных анизотропий двух слоев Со [6] и интерфейсным DMI [7]. Мы оценили вклад разницы поверхностных анизотропий слоев Со в разницу частот пиков Стокса и анти-Стокса. Поверхностная анизотропия нижних слоев Со у всех образцов была одинакова, так как у них одинаковая толщина $(1.1 \mathrm{~nm})$ и одинаковые покровные слои $\mathrm{Pt}(3.2 \mathrm{~nm})$ и $\operatorname{Ir}(1.4 \mathrm{~nm})$. Поверхностная константа анизотропии была рассчитана из соотношения $K_{\mathrm{S}}=K \cdot t_{\mathrm{FM}}$, где $K-$ константа магнитокристаллической анизотропии, $t_{\mathrm{FM}}-$ толщина ферромагнитного слоя. Константа магнитокристаллической анизотропии рассчитывается из соотношения $K=\left(H_{m} \cdot M_{\mathrm{S}}\right) / 2$, где $H_{m}=H_{A}+4 \pi M_{\mathrm{S}}-$ поле магнитокристаллической анизотропии, $H_{A}$ - эффективное поле магнитной анизотропии. Эффективное поле анизотропии $H_{A}$ равно полю, в котором пересекаются зависимости $M(H)$, записанные при двух ориентациях магнитного поля вдоль легкой оси намагничивания и вдоль трудной оси намагничивания (рис. 2). Константа поверхностной анизотропии нижнего слоя $K_{\mathrm{S} 1}=0.95 \mathrm{erg} / \mathrm{cm}^{2}$. Константы поверхностной анизотропии верхних слоев Со были следующими: для $t_{\mathrm{Co}}=0.6 \mathrm{~nm} \quad K_{\mathrm{S} 2}=0.52 \mathrm{erg} / \mathrm{cm}^{2}$; для $t_{\mathrm{Co}}=0.7 \mathrm{~nm}$ $K_{\mathrm{S} 2}=0.6 \mathrm{erg} / \mathrm{cm}^{2}$; для $t_{\mathrm{Co}}=0.8 \mathrm{~nm} K_{\mathrm{S} 2}=0.69 \mathrm{erg} / \mathrm{cm}^{2}$ и для $t_{\mathrm{Co}}=1.0 \mathrm{~nm} K_{\mathrm{S} 2}=0.86 \mathrm{erg} / \mathrm{cm}^{2}$. Разница частот пиков МБС, вызванная разницей поверхностных анизотропий рассчитывается по формуле [6]:

$$
\Delta f=\frac{8 \gamma}{\pi^{3}} \frac{K_{\mathrm{S} 1}-K_{\mathrm{S} 2}}{M_{\mathrm{S}}} \frac{k_{\mathrm{x}}}{1+l_{\mathrm{ex}}^{2} \pi^{2} / t_{\mathrm{FM}}^{2}},
$$

где $K_{\mathrm{S} 1}$ и $K_{\mathrm{S} 2}-$ константы поверхностной анизотропии закрепленного и свободного слоев $\mathrm{Co}, k_{\mathrm{x}}-$ проекция волнового вектора падающего света на ось $x, t_{\mathrm{FM}}-$ толщина ферромагнитного слоя, $l_{\mathrm{ex}}=\left(2 A / 4 \pi M_{\mathrm{S}}^{2}\right)^{1 / 2}-$ обменная длина. Разница частот пиков МБС, рассчитанная из (1), составляет $0.47-2.27 \mathrm{MHz}$. Этот сдвиг мал по сравнению с наблюдаемой в эксперименте разницей частот в $\Delta f\left(k_{\mathrm{x}}\right) \sim 1-3 \mathrm{GHz}$. Таким образом, основной вклад в разницу частот вносит поверхностное DMI.

Формально закон дисперсии спиновых волн для одиночной пленки $[4,7]$ позволяет приближенно определить плотность энергии DMI и в двуслойной структуре

$$
\begin{aligned}
& 2 \pi f=\gamma\left(H_{\mathrm{IP}}+J k_{\mathrm{x}}^{2}+\xi\left(k_{\mathrm{x}} L\right) M_{\mathrm{S}}\right)^{1 / 2} \\
& \times\left(H_{\mathrm{IP}}-H_{\mathrm{A}}+J k_{\mathrm{x}}^{2}+M_{\mathrm{S}}-\xi\left(k_{\mathrm{x}} L\right) M_{\mathrm{S}}\right)^{1 / 2}-\frac{2 \gamma}{M_{\mathrm{S}}} D k_{\mathrm{x}},
\end{aligned}
$$

где $H_{\mathrm{A}}=10 \mathrm{kOe}-$ эффективное поле магнитной анизотропии, определенное из данных СКВИД-магнитометрии (см. рис. 2), $J=2 A / M_{\mathrm{S}}-$ обменная константа, $L$ - толщина ферромагнитного слоя, $\xi\left(k_{\mathrm{x}} L\right)=1-\left(1-\exp \left(-\left|k_{\mathrm{x}} L\right|\right)\right) /\left|k_{\mathrm{x}} L\right|$. Из закона дисперсии следует, что $D$ зависит от разницы частот $\Delta f$ в соответствии с формулой

$$
D=\frac{\Delta f \pi M_{\mathrm{S}}}{\gamma k_{\mathrm{x}}}
$$

Уравнением (3) были определены значения $D$ для всех четырех образцов серии (рис. 6). Из рис. 6 видно, что с увеличением толщины верхнего слоя Со, величина $D$ уменьшается, как в случае одиночной пленкой Со [14,29-31]. Из литературы известно, что наблюдается обратно пропорциональная зависимость $D \sim 1 / t_{\text {Со }}$ с ро-

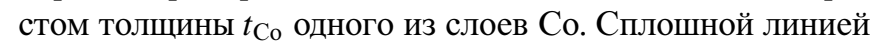
на рис. 6 показана аппроксимация экспериментальных данных гиперболой.

Для образцов на основе одиночного слоя Со [11-14] и для образцов на основе множества несвязанных обменом слоев Со одинаковой толщины, величина $D$ ожидается одинаковой. Величины $D$ исследуемых синтетических ферримагнетиков $\left(1.7-2.4 \mathrm{erg} / \mathrm{cm}^{2}\right)$ больше значений $D$ 

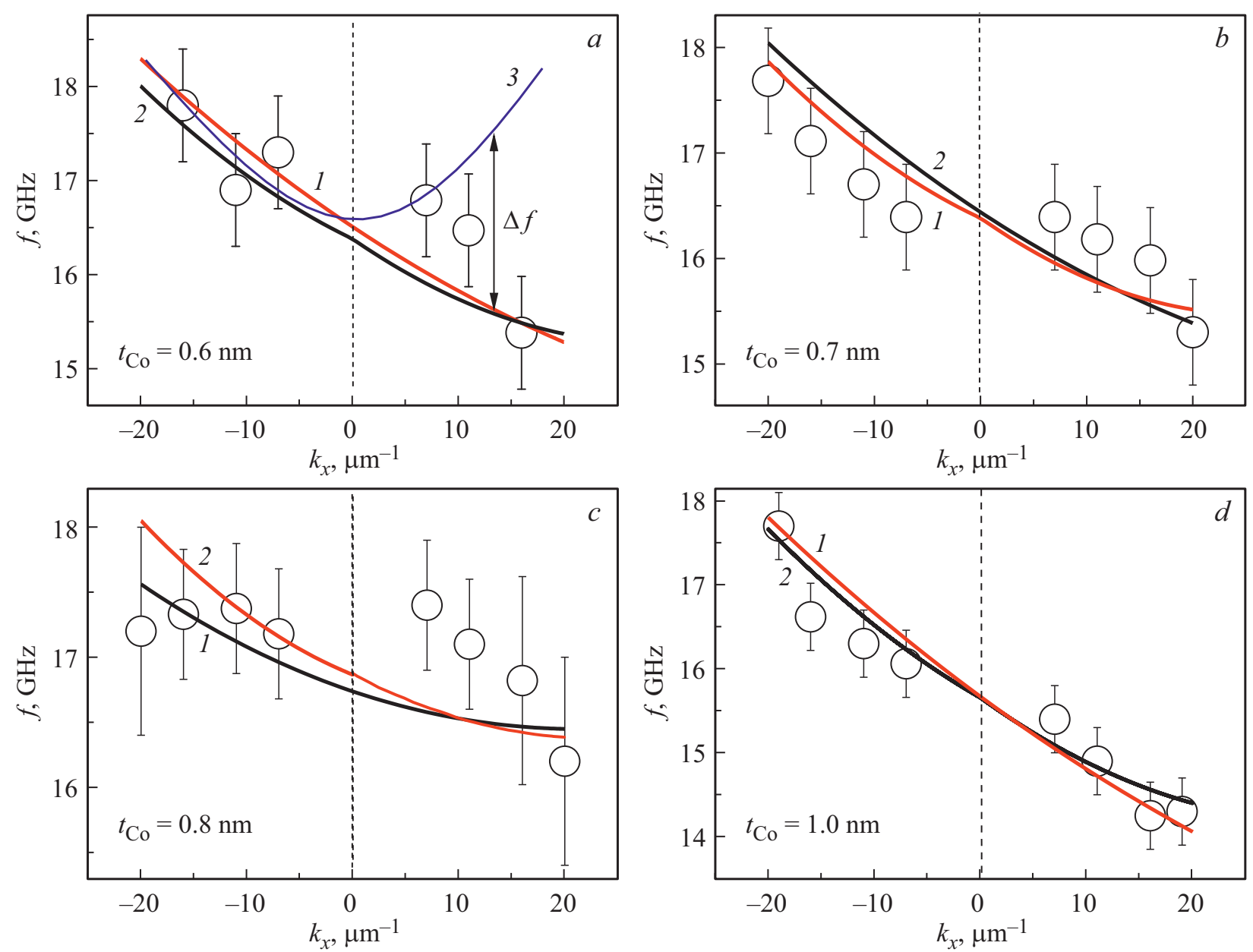

Рис. 5. Зависимости частоты спиновых волн $f$, записанных в постоянном магнитном поле $H_{\mathrm{IP}}=+8 \mathrm{kOе}$, от проекции волнового вектора лазера $k_{\mathrm{x}}$ на ось $x$ для образцов с $(a) t_{\mathrm{Co}}=0.6 \mathrm{~nm},(b) t_{\mathrm{Co}}=0.7 \mathrm{~nm},(c) t_{\mathrm{Co}}=0.8 \mathrm{~nm},(d) t_{\text {Со }}=1.0 \mathrm{~nm}$. Сплошная линия $1-$ аппроксимация моделью [14], сплошная линия $2-$ аппроксимация уравнением (2). Сплошная линия 3 на рис. $5, a-$ дисперсия $f\left(k_{\mathrm{x}}\right)$ в отсутствие DMI.

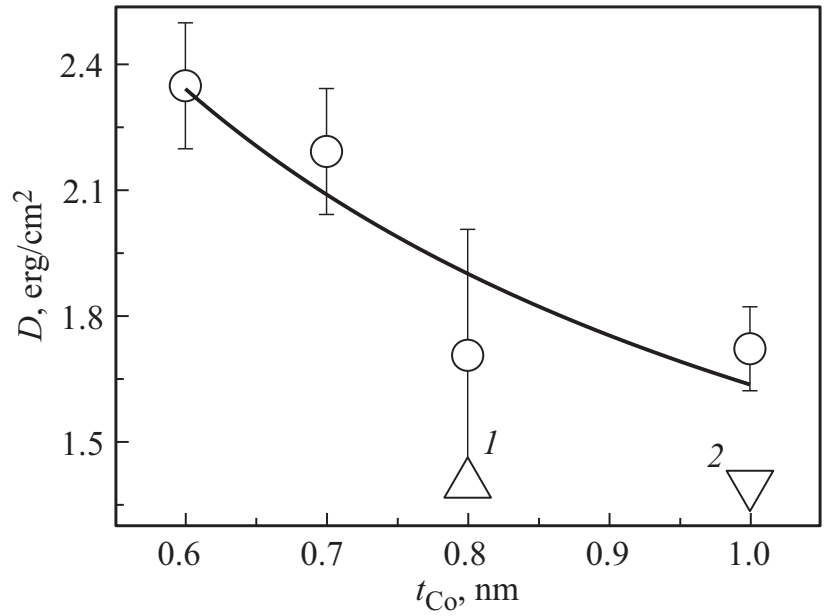

Рис. 6. Зависимость плотности энергии интерфейсного DMI

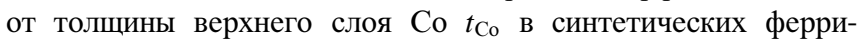
магнетиках $\operatorname{Pt}(3.2 \mathrm{~nm}) / \mathrm{Co}(1.1 \mathrm{~nm}) / \mathrm{Ir}(1.4 \mathrm{~nm}) / \mathrm{Co}\left(t_{\mathrm{Co}}\right) / \mathrm{Pt}(3.2 \mathrm{~nm})$. Точка 1 значение $D$ для гетероструктуры $\mathrm{Pt} / \mathrm{Co}(0.8 \mathrm{~nm}) / \mathrm{Ir}[20]$, 2 - значение $D$ для гетероструктуры $\mathrm{Pt} / \mathrm{Co}(1.0 \mathrm{~nm}) / \mathrm{Ir}[29]$. Сплошной линией показана аппроксимация гиперболой. для одиночных слоев Со, которые показаны на рис. 6 точками 1 и 2 и равны $1.4 \mathrm{erg} / \mathrm{cm}^{2}$. Увеличение $D$ по сравнению с однослойным образцом может быть вызвано несколькими факторами: 1) ростом числа интерфейсов Pt/Co и Ir/Co, на которых возникает DMI, 2) уменьшением шероховатости интерфейсов, 3) межслоевым обменным взаимодействием, которое делает DMI в двух слоях Со не независимым друг от друга, а увеличивает его в результате переноса спиновой поляризации между слоями.

Если пренебречь межслойным обменным взаимодействием нельзя, нужно использовать более сложный закон дисперсии спиновых волн $f\left(k_{\mathrm{x}}\right)$ [4], учитывающий величину и знак обменного взаимодействия двух антиферромагнитно связанных слоев. Для описания закона дисперсии спиновых волн синтетического ферримагнетика была использована модель из [20]. Она не учитывает разницу частот $\Delta f$, вызванную DMI, но учитывает межслоевое обменное взаимодействие между слоями Сo. Для аппроксимации дисперсии спиновых волн мо- 
делью из [20] мы добавили к решению уравнения $f\left(k_{\mathrm{x}}\right)$ разницу частот пиков Стокса и анти-Стокса, вызванную DMI $\Delta f=\gamma D k_{\mathrm{x}} / \pi M_{\mathrm{S}}$, так же, как это делалось для однослойного образца в формуле (3). Результат аппроксимации модифицированными формулами [20] показан на рис. $5, a-d$ сплошными линиями 1 . Для сравнения мы использовали закон дисперсии спиновых волн (2), не учитывающий антиферромагнитное обменное взаимодействия между слоями Со. Он показан на рис. $5, a-d$ сплошными линиями 2. Оба используемых закона удовлетворительно описывают экспериментальные данные. Сильное расхождение экспериментальных данных и аппроксимаций для образца с $t_{\mathrm{Co}}=0.8 \mathrm{~nm}$ вызвано погрешностью измерений. Таким образом, закон дисперсии спиновых волн, описанный в [4], может использоваться для аппроксимации зависимостей $f\left(k_{\mathrm{x}}\right)$ синтетических антиферромагнетиков и ферримагнетиков и позволяет оценивать величину взаимодействия ДзялошинскогоМория в них.

\section{4. Заключение}

В синтетических ферримагнетиках с перпендикулярной магнитной анизотропией обнаружены акустические и оптические моды спиновых волн. Был оценен вклад поверхностной анизотропии и DMI в разницу частот пиков Стокса и анти- Стокса оптической моды. Основной вклад в разницу частот вносит интерфейсное DMI. При увеличении толщины верхнего слоя Со синтетического ферримагнетика $\mathrm{Pt} / \mathrm{Co} / \mathrm{Ir} / \mathrm{Co} / \mathrm{Pt}$ его значение плотности энергии DMI уменьшается линейно, как в гетероструктурах с одним слоем Со. Измеренные значения плотности энергии DMI $D$ двух обменно-связанных слоев Со больше значений $D$ одиночных слоев Со. Это может быть вызвано увеличением количества интерфейсов, на которых возникает DMI, шероховатостью интерфейсов и наличием обменного межслоевого взаимодействия, за счет которого отдельные слои Со вносят вклад в суммарное DMI. Закон дисперсии спиновых волн для одиночных ферромагнитных пленок качественно и количественно описывает дисперсию в гетероструктурах, состоящих из двух слоев Со, связанных антиферромагнитным обменным взаимодействием.

\section{Финансирование работы}

Работа выполнена в рамках темы AАAА-A19119092390079-8 госзадания ИПХФ РАН. Р.Б. Моргунов поддержан грантом Президента Российской Федерации для ведущих научных школ 2644.2020.2. А.И. Безверхний поддержан грантом РФФИ в рамках научного проекта № 19-32-90128. А.В. Садовников и В.А Губанов поддержаны грантом РФФИ в рамках научного проекта № 18-29-27026.

\section{Конфликт интересов}

Авторы заявляют, что у них нет конфликта интересов.

\section{Список литературы}

[1] A. Fert, F. Nguyen Van Dau. Physique 20, 817 (2019).

[2] P. Grünberg, R. Schreiber, Y. Pang. Phys. Rev. Lett. 57, 2442 (1986).

[3] J. Barnas, P. Grünberg. J. Magn. Magn. Mater 82, 186 (1986).

[4] K. Di, V.L. Zhang, H.S. Lim, S. Ch. Ng. Phys. Rev. Lett. 114, 047201 (2015).

[5] J.J. Krebs, P. Lubitz, A. Chaiken, G.A. Prinz. Phys. Rev. Lett. 63, 1645 (1989).

[6] S.M. Chérif, Y. Roussigné, P. Moch. J. Appl. Phys. 98, 063905 (2005).

[7] J. Cho, N.-H. Kim, S. Lee, J.-S. Kim, R. Lavrijsen, A. Solignac, Y. Yin, D.-S. Han, N.J.J. van Hoof, H.J.M. Swagten, B. Koopmans, Ch.Y. You. Nature Commun. 6, 7635 (2015).

[8] P.M. Levy, A. Fert. Phys. Rev. B 23, 4667 (1981).

[9] Y. Roussigné, F. Ganot, C. Dugautier, P. Moch. Phys. Rev. B 52, 350 (1995).

[10] J. Fassbender, F. Nörtemann, R.L. Stamps, R.E. Camley, B. Hillebrands, G. Güntherodt, S.S.P. Parkin. Phys. Rev. B 46, 5810 (1992).

[11] S.-G. Je, D.-H. Kim, S.-C. Yoo, B.-C. Min, K.-J. Lee, S.-B. Choe. Phys. Rev. B 88, 214401 (2013).

[12] R. Lavrijsen, D.M.F. Hartmann, A. van den Brink, Y. Yin, B. Barcones, R.A. Duine, M.A. Verheijen, H.J.M. Swagten, B. Koopmans. Phys. Rev. B 91, 104414 (2015).

[13] A. Hrabec, N.A. Porter, A. Wells, M.J. Benitez, G. Burnell, S. Mc Vitie, D. Mc Grouther, T.A. Moore, C.H. Marrows. Phys. Rev. B. 90, 020402(R) (2014).

[14] K. Shahbazi, J.-V. Kim, H.T. Nembach, J.M. Shaw, A. Bischof, M.D. Rossel, V. Jeudy, T.A. More, C.H. Marrows. Phys. Rev. B 99, 094409 (2019).

[15] N. Nakajima, T. Koide, T. Shidara, H. Miyauchi, H. Fukutani, A. Fujimori, K. Iio, T. Katayama, M. Nývlt, Y. Suzuki. Phys. Rev. Lett. 81, 5229 (1998).

[16] R. Morgunov, A. Hamadeh, T. Fache, G. Lvova, O. Koplak, A. Talantsev, S. Mangin. Superlat. Microstruct. 104, 509 (2017).

[17] R.B. Morgunov, A.V. Yurov, V.A. Yurov, A.D. Talantsev, A.I. Bezverhnii, O.V. Koplak. Phys. Rev. B 100, 144407 (2019).

[18] E.R. Moog, S.D. Bader, J. Zak. App. Phys. Lett. 56, 2687 (1990).

[19] K. Zakeri. Phys. Rep. 545, 47 (2014).

[20] S.M. Rezende, C. Chesman, M.A. Lucena, A. Azevedo, F.M. de Aguiar. J. Appl. Phys. 54, 958 (1998).

[21] B. Hillebrands. Phys. Rev. B 41, 530 (1990).

[22] A. Haldar, C. Banerjee, P. Laha, A. Barman. J. Appl. Phys. 115, 133901 (2014).

[23] N. Nozawa, S. Saito, S. Hinata, M. Takahasi. J. Phys. D 46, 172001 (2013).

[24] M. Belmeguenai, J.-P. Adam, Y. Roussigne, S. Eimer, T. Devolder, J.-V. Kim, S.M. Chérif, A. Stashkevich, A. Thiaville. Phys. Rev. B 91, 180405(R) (2015). 
[25] M. Belmeguenai, H. Bouloussa, Y. Roussigné, M.S. Gabor, T. Petrisor, Jr., C. Tiusan, H. Yang, A. Stashkevich, S.M. Chérif. Phys. Rev. B 96, 144402 (2017).

[26] N.A. Sergeeva, S.M. Chérif, A.A. Stashkevichm, M.P. Kostylev, J. Ben Yussef. J. Magn. Magn. Mater 288, 250 (2005).

[27] I.B.-El Mokhtari, D. Ourdani, Y. Roussigné, R.B. Mos, M. Nasui, F. Kail, L. Chahed, S.M. Chérif, A. Stashkevich, M. Gabor, M. Belmeguenai. J. Phys.: Condens. Matter. 32, $495802(2020)$.

[28] L.M. Li, B.-Z. Li, F.-C. Pu. J. Phys.: Condens. Matter 6, 1941 (2994).

[29] M. Belmeguenai, Y. Roussigné, S.M. Chérif, A. Stashkevich, T. Petrisor jr., M. Nasui, M.S. Gabor. J. Phys. D 52, 125002 (2019).

[30] N.-H. Kim, J. Jung, J. Cho, D.-S. Han, Y. Yin, J.-S. Kim, H.J.M. Swagten, Ch.-Y. You. Appl. Phys. Lett. 108, 142406 (2016).

[31] H.T. Nembach, J.M. Shaw, M. Weiler, E.J. Thomas, J. Silvia. Nature Phys. 11, 825 (2015).

Редактор Т.Н. Василевская 